\title{
Indeterminate Pulmonary Nodules at Diagnosis in Rhabdomyosarcoma: Are They Clinically Significant? A Report From the European Paediatric Soft Tissue Sarcoma Study Group
}

Bas Vaarwerk, MD ${ }^{1,10}$; Gianni Bisogno, MD, PhD²; Kieran McHugh, MD³; Hervé J. Brisse, MD, PhD ${ }^{4}$; Carlo Morosi, MD ${ }^{5}$; Nadège Corradini, MD ${ }^{6}$; Meriel Jenney, MD7; Daniel Orbach, MD ; Julia C. Chisholm, MD, PhD ${ }^{8}$; Andrea Ferrari, MD ${ }^{5}$; Ilaria Zanetti²; Gian Luca De Salvo, MD ${ }^{9}$; Rick R. van Rijn, MD, PhD ${ }^{1}$; and Johannes H.M. Merks, MD, PhD ${ }^{1,10}$ on behalf of the EpSSG Radiology Group

PURPOSE To evaluate the clinical significance of indeterminate pulmonary nodules at diagnosis (defined as $\leq 4$ pulmonary nodules $<5 \mathrm{~mm}$ or 1 nodule measuring $\geq 5$ and $<10 \mathrm{~mm}$ ) in patients with pediatric rhabdomyosarcoma (RMS).

PATIENTS AND METHODS We selected patients with supposed nonmetastatic RMS treated in large pediatric oncology centers in the United Kingdom, France, Italy, and the Netherlands, who were enrolled in the European Soft Tissue Sarcoma Study Group (EpSSG) RMS 2005 study. Patients included in the current study received a diagnosis between September 2005 and December 2013, and had chest computed tomography scans available for review that were done at time of diagnosis. Local radiologists were asked to review the chest computed tomography scans for the presence of pulmonary nodules and to record their findings on a standardized case report form. In the EpSSG RMS 2005 Study, patients with indeterminate pulmonary nodules were treated identically to patients without pulmonary nodules, enabling us to compare event-free survival and overall survival between groups by log-rank test.

RESULTS In total, 316 patients were included; 67 patients (21.2\%) had indeterminate pulmonary nodules on imaging and 249 patients (78.8\%) had no pulmonary nodules evident at diagnosis. Median follow-up for survivors ( $n=258$ ) was 75.1 months; respective 5 -year event-free survival and overall survival rates $(95 \% \mathrm{Cl}$ ) were $77.0 \%(64.8 \%$ to $85.5 \%)$ and $82.0 \%(69.7 \%$ to $89.6 \%)$ for patients with indeterminate nodules and $73.2 \%(67.1 \%$ to $78.3 \%)$ and $80.8 \%(75.1 \%$ to $85.3 \%)$ for patients without nodules at diagnosis $(P=.68$ and .76, respectively).

CONCLUSION Our study demonstrated that indeterminate pulmonary nodules at diagnosis do not affect outcome in patients with otherwise localized RMS. There is no need to biopsy or upstage patients with RMS who have indeterminate pulmonary nodules at diagnosis.

J Clin Oncol 37:723-730. ๑ 2019 by American Society of Clinical Oncology

ASSOCIATED

CONTENT

Appendix

Data Supplement

Podcast by Dr Pappo

Author affiliations

and support

information (if

applicable) appear

at the end of this

article.

Accepted on

November 28, 2018 and published at jco. org on January 31 ,

2019: DOI https://doi.

org/10.1200/JCO.18.

01535

\section{INTRODUCTION}

Over the past decades, the 5-year overall survival (OS) for patients with nonmetastatic rhabdomyosarcoma (RMS) has improved to approximately $80 \%{ }^{1-3} \mathrm{Nev}$ ertheless, survival for patients with metastatic disease remains poor, with 3-year OS ranging between 34\% and $56 \% .4,5$ The lungs are the most frequently involved metastatic site and patients with only pulmonary metastases have a better prognosis than patients with metastases located outside the lungs. Nevertheless, accurate staging of the lungs is important to select patients who require chest radiotherapy and additional chemotherapy. Staging for lung metastases is usually done by chest computed tomography (CT). Improved quality and increased spatial resolution chest $\mathrm{CT}$ scans have introduced new diagnostic dilemmas, because smaller nodules also became detectable. Small subcentimeter pulmonary nodules are a frequent normal finding in healthy children; however, differentiation between small metastatic and benign nodules is difficult or even impossible in children with extrathoracic malignancies. ${ }^{6-12}$ Because of the size of these small nodules, percutaneous needle biopsy is usually not feasible and the decision to treat patients according to nonmetastatic or metastatic guidelines is based, therefore, on the characteristics and number of nodules seen on chest CT imaging. Among other parameters, radiologists use nodule size, margins, the presence of calcification, and the total number of nodules to estimate the likelihood that the nodules 
represent metastases. However, none of these characteristics adequately distinguishes malignant from benign lesions. ${ }^{7,9,10}$

In the European Pediatric Soft Tissue Sarcoma Study Group (EpSSG) RMS 2005 protocol, patients with no more than four pulmonary nodules of less than $5 \mathrm{~mm}$ or one nodule measuring between 5 and less than $10 \mathrm{~mm}$ were considered to have indeterminate or equivocal lesions. The assumption was made that some of these nodules were benign lesions and others were micrometastases, which, in the past, were not visible because of the use of chest radiographs. Because the impact of these micrometastases on survival was unclear, it was decided by the EpSSG protocol committee that patients classified as having indeterminate pulmonary lesions should be treated as those with localized disease.

If this assumption is wrong, survival may be impaired for this patient group and, consequently, these patients should be upstaged to a higher risk category with intensified treatment in future protocols. Therefore, the aim of this study was to assess the clinical significance of indeterminate pulmonary nodules at diagnosis in children with otherwise nonmetastatic RMS, by comparing eventfree survival (EFS) and OS for patients with indeterminate pulmonary nodules to those without such lesions (ie, lungs entirely clear on CT scans).

\section{PATIENTS AND METHODS}

Patients included in this analysis were those enrolled in the EpSSG RMS 2005 study (EudraCT no: 2005-000217-35) for nonmetastatic RMS and for whom the diagnosis was confirmed by central pathology review and whose chest CT scan at diagnosis was available for radiologic review. Informed consent had been obtained from the patient or guardian or both, according to the research ethics requirements of the individual institutions. Included patients received a diagnosis between September 2005 and December 2013 to allow adequate follow-up. Patients in whom indeterminate pulmonary nodules had been biopsied were excluded.

For the current analysis, we invited local radiologists from larger pediatric oncology centers to review the chest CT scans at diagnosis for patients with localized disease diagnosed in their center (Fig 1). Eligible patients were recruited in 12 larger pediatric oncology centers in France (Institut Curie, Paris; and Centre Léon Bérard, Lyon), Italy (Istituto Nazionale Tumori Milano; and Padova University Hospital), the Netherlands (Beatrix Children's HospitalUniversity Medical Center Groningen; and Emma Children's Hospital-Academic Medical Center), and the United Kingdom (Birmingham's Children's Hospital; Bristol Royal Hospital for Children; Children's Hospital for Wales; Great Ormond Street Hospital for Children; Royal Manchester Children's Hospital; and Royal Marsden Hospital).
The outline of the randomized part of the EpSSG RMS 2005 study has been described previously. ${ }^{3}$ Treatment was stratified according to risk group on the basis of pathology, postsurgical stage (IRS group), site, nodal involvement, size, and age (Data Supplement). In general, all patients received multidrug chemotherapy comprising ifosfamide (except for low-risk patients), vincristine, and dactinomycin (IVA). High-risk patients were randomly assigned to either nine courses of standard IVA therapy or IVA with doxorubicin. The results of this randomization did not show a difference in survival between the treatment arms. ${ }^{3}$ After nine courses of chemotherapy, high-risk patients in clinical complete remission were eligible for a second randomization between end of therapy (standard) and six courses (4 weeks each) of metronomic maintenance therapy with vinorelbine and cyclophosphamide. Patients at very high risk (ie, with alveolar histology and positive regional lymph nodes) received IVA with doxorubicin, followed by standard maintenance therapy with vinorelbine and cyclophosphamide. ${ }^{13}$

Local primary therapy was determined by risk group, tumor site, age of patient, and response assessment. Delayed surgery, on the basis of resectability without mutilating consequences, was performed for residual tumor. If recommended, radiotherapy was given at week 13 . Radiation doses ranged between 36 and 50.4 Gy, depending on histology, resection margins, and tumor response.

Central radiology review was not part of the EpSSG-RMS 2005 protocol; for the current analysis, all chest CT scans at diagnosis were reviewed by the local radiologist in the treating centers for the presence of indeterminate pulmonary nodules. Data were recorded using a standardized case report form to enhance uniformity among the radiologists. According to protocol, chest CT scans were performed with a minimum reconstruction slice width of 3 to $5 \mathrm{~mm}$

Scanning parameters and number and size of nodules were noted. Patients were classified as having no nodules, indeterminate pulmonary nodules, or misclassified as indeterminate lesions. Indeterminate pulmonary nodules, according to the EpSSG RMS 2005 protocol, were defined as no more than four nodules of less than $5 \mathrm{~mm}$ or one nodule measuring between $5 \mathrm{~mm}$ and less than $10 \mathrm{~mm}$. Patients with pulmonary nodules fulfilling definitions of pulmonary metastases were categorized as having nodules misclassified as indeterminate lesions and excluded from the current analysis $(n=2)$.

\section{STATISTICAL ANALYSES}

Analyses were performed using SAS, version 9.4 (SAS Institute, Cary, NC). Data from the reviews of the chest CT scans were combined with treatment and outcome data from the EpSSG database. The distribution of patient characteristics between patients with indeterminate 


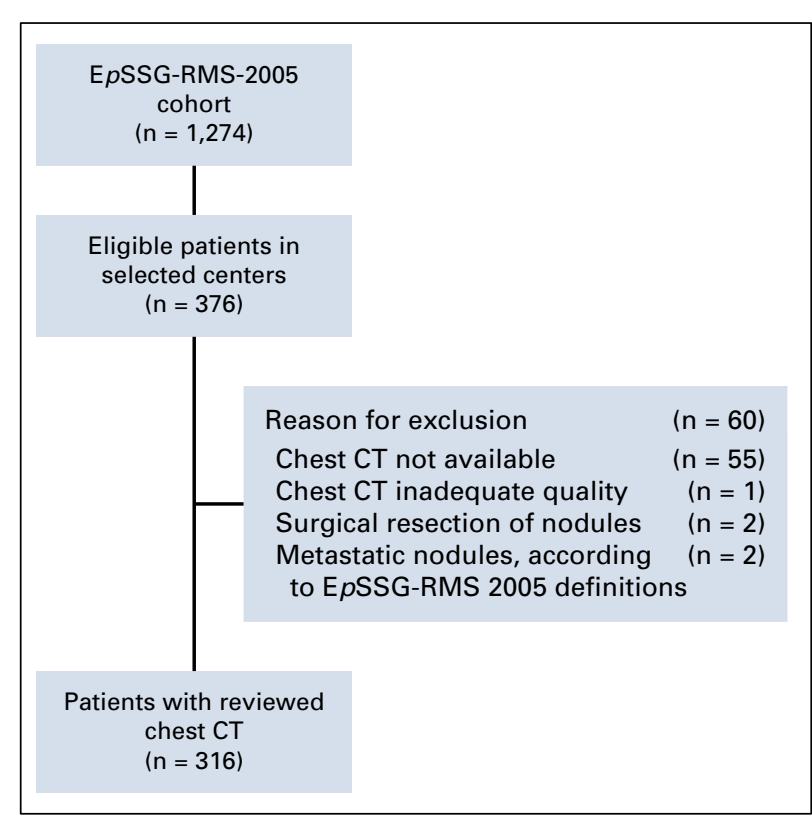

FIG 1. Flow diagram for the current analysis. CT, computed tomography; EpSSG, European Soft Tissue Sarcoma Study Group; RMS, rhabdomyosarcoma.

pulmonary nodules at diagnosis and patients without pulmonary nodules was compared using $\chi^{2}$ tests. OS was calculated from the date of diagnosis to death from any cause, and EFS was measured from the date of diagnosis to disease progression, relapse, a second malignancy, or death from any cause. Outcomes for living patients were censored at the time of their last reported contact. EFS and OS curves were obtained using the Kaplan-Meier method (data cutoff point was November 1, 2017). ${ }^{14}$ A log-rank test was used to compare the EFS and OS levels between the patients with indeterminate pulmonary nodules and patients without pulmonary nodules at diagnosis. Subgroup analyses were performed on the basis of histology, fusion status, age at diagnosis, and received therapy.,15,16 $P$ less than .05 was considered statistically significant.

\section{RESULTS}

\section{Patients}

In total, 376 eligible patients were enrolled in the EpSSG RMS 2005 study for localized disease. The primary reason for exclusion was that the chest CT scan at diagnosis was not available for review $(n=55)$. Patients were also excluded because they had a surgical resection of pulmonary nodules $(n=2)$, radiologic review showed pulmonary nodules considered metastatic $(n=2)$, or the chest CT scan had a slice thickness greater than $5 \mathrm{~mm}$, considered inappropriate to determine the presence of small pulmonary nodules $(n=1)$. Eventually, data from 316 patients were available for analysis (Fig 1). Clinical characteristics for the included patients were comparable to the total group of eligible patients. CT slice thickness was no greater than
$3 \mathrm{~mm}$ in 214 of 316 of the included patients (67.7\%) and the reconstruction width was no greater than $1.25 \mathrm{~mm}$ in 77 of 316 patients (24.4\%). Median age at diagnosis was 5.4 (the range was 0 to 21.9) years, and the median follow-up time for survivors was 75.1 (interquartile range was 54.4 to 94.6) months.

The majority of patients (80.7\%) had an Intergroup Rhabdomyosarcoma Study Group III (IRS group III) tumor at diagnosis (ie, incompletely resected tumor/biopsy only) and specimens of $70.9 \%$ of the patients showed favorable histology. All patients received chemotherapy according to protocol. In total, 77 patients (24\%) received maintenance chemotherapy. Most patients (77\%) received local radiotherapy and 135 of 255 IRS group III patients (53\%) underwent secondary surgery. Patients' and treatment characteristics are further described in Table 1 and in the Data Supplement. Compared with the total EpSSG RMS 2005 cohort, within this subgroup with reviewed chest CT scans, there were significantly more IRS group III and highrisk patients ( $P=.01$; Data Supplement).

\section{Nodule Characteristics}

In total, 249 patients (78.8\%) did not have pulmonary nodules at diagnosis; 67 of the 316 patients $(21.2 \%$ ) had at least one indeterminate pulmonary nodule. Patient and treatment characteristics were comparable for patients with indeterminate nodules and patients without nodules (Table 1). A total of 100 nodules were observed in 67 patients, 46 of whom (68.7\%) had only one nodule. The size of the nodules ranged from 1 to $8 \mathrm{~mm}$ and in 37 of the 67 patients $(55.2 \%)$, the largest nodule was 1 to $2 \mathrm{~mm}$ (Table 2).

\section{Indeterminate Nodules and Impact on Survival}

Five-year EFS was $77.0 \%$ (95\% Cl: $64.8 \%$ to $85.5 \%$ ) for patients with indeterminate nodules and $73.2 \%(95 \% \mathrm{Cl}$ : $67.1 \%$ to $78.3 \%)$ for patients without nodules ( $P=.68$ ). Five-year OS was $82.0 \%$ (95\% Cl: $69.7 \%$ to $89.6 \%$ ) for patients with indeterminate pulmonary nodules and $80.8 \%$ (95\% Cl: $75.1 \%$ to $85.3 \%$ ) for patients without nodules ( $P=$ .76). No significant differences in EFS and OS were found on the basis of the presence of indeterminate pulmonary nodules (Fig 2) or on the basis of the number and size of the largest nodule (Table 3). Subgroup analyses according to histology, fusion status, age at diagnosis, and received chemotherapy regimen (with or without doxorubicin or with or without maintenance chemotherapy) showed no significant differences in EFS and OS based on the presence of indeterminate nodules.

Eighty-three patients experienced at least one event; 67 patients $(80.7 \%)$ had no pulmonary nodules at diagnosis and 16 patients (19.3\%) had at least one indeterminate pulmonary nodule at diagnosis. First relapse was locoregional in 64 patients $(77.1 \%)$, only metastatic in eight patients (9.6\%), and combined locoregional and metastatic in seven patients (8.4\%). Four patients developed a second 
TABLE 1. Patients' and Tumor Characteristics at Diagnosis Based on Presence of Indeterminate Pulmonary Nodules

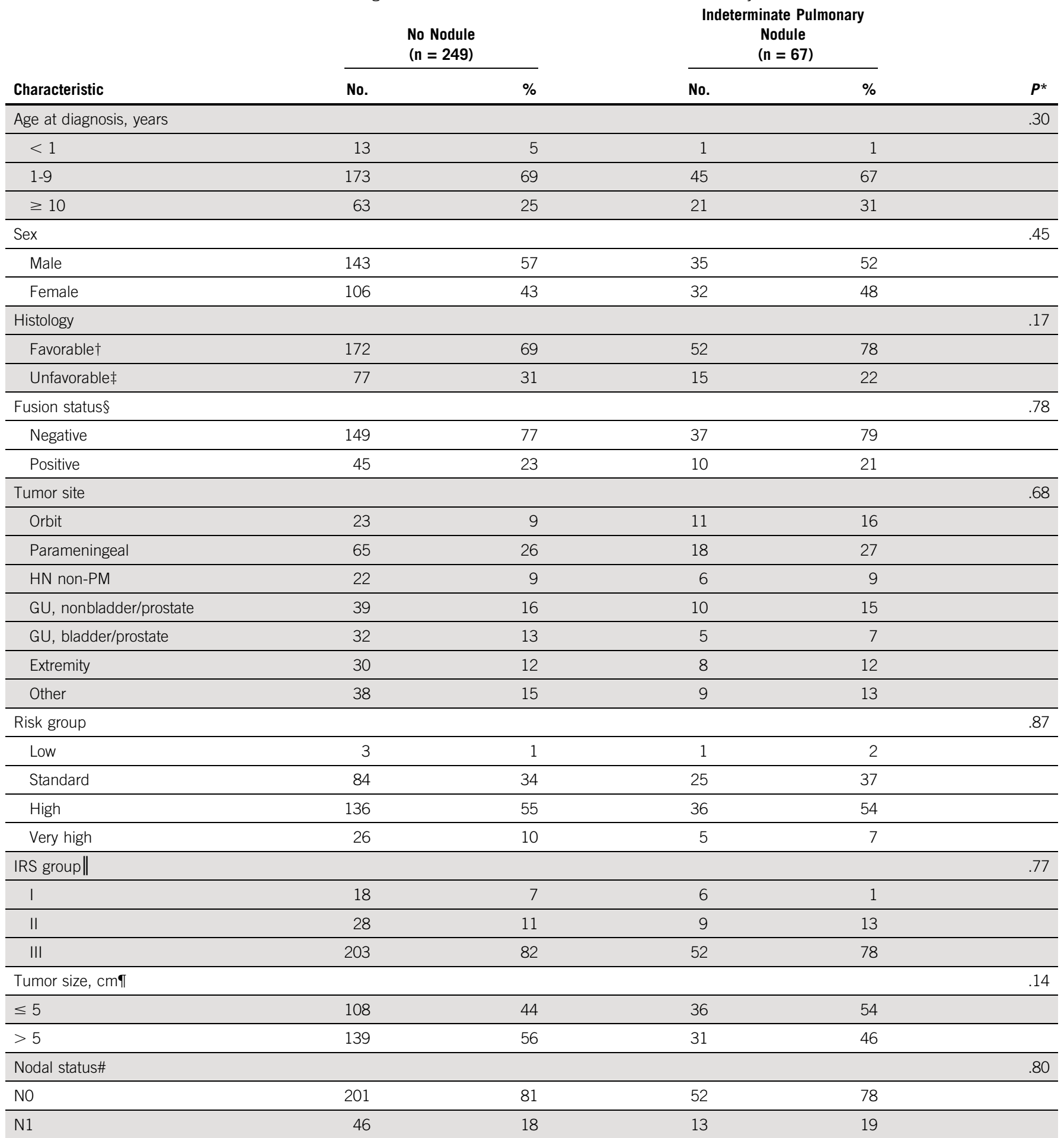

Abbreviations: GU, genitourinary; HN non-PM, head-neck nonparameningeal.

*Based on $\chi^{2}$ test.

†All embryonal, spindle-cell, botryoid rhabdomyosarcoma.

$\ddagger$ Unfavorable results are all from alveolar rhabdomyosarcoma.

§Fusion status was not investigated in 75 patients (no pulmonary nodules, $n=55$; indeterminate pulmonary nodules, $n=20$ ).

\|IRS (Intergroup Rhabdomyosarcoma Study) Group I, primary complete resection (R0); group II, microscopic residual (R1) or primary complete resection but N1; group III, macroscopic residual (R2).

ITumor size was unknown in two patients (no pulmonary nodules).

\#Nodal status was unknown in four patients (no pulmonary nodules, $n=2$; indeterminate pulmonary nodules, $n=2$ ). 
TABLE 2. Characteristics of Indeterminate Pulmonary Nodules in 67 Patients Characteristic

No.

$\%$

\begin{tabular}{crr}
\hline No. of nodules & & \\
\hline 1 & 46 & 69 \\
\hline 2 & 13 & 19 \\
\hline 3 & 4 & 6 \\
\hline 4 & 4 & 6 \\
\hline
\end{tabular}

Nodule maximum diameter, $\mathrm{mm}$

\begin{tabular}{ccr}
\hline 1 & 13 & 19 \\
\hline 2 & 24 & 36 \\
\hline 3 & 15 & 22 \\
\hline 4 & 10 & 15 \\
\hline 5 & 3 & 4 \\
\hline 7 & 1 & 1 \\
\hline 8 & 1 & 1 \\
\hline Laterality & & \\
\hline Unilateral & 57 & 85 \\
\hline Bilateral & 10 & 15
\end{tabular}

malignancy (no tumor predisposition syndromes were reported for these patients). In the group of 67 patients with indeterminate pulmonary nodules, lung metastases developed in two (3.0\%), compared with four of 249 patients $(1.6 \%)$ in the group without nodules $(P=.46$; Table 4$)$.

\section{DISCUSSION}

Small pulmonary nodules at time of diagnosis are a diagnostic challenge in children with RMS. The results of this study confirm that the presence of indeterminate pulmonary nodules is a frequently encountered diagnostic problem. More importantly, the results of this study demonstrate that the presence of indeterminate pulmonary nodules at diagnosis does not affect survival for patients treated according to EpSSG guidelines for localized disease.

The incidence of pulmonary nodules in our cohort was lower than reported in nononcologic populations (up to $38 \%) .{ }^{11,12}$ This difference might be explained by variability in CT slice reconstruction methods. In the EpSSG RMS 2005 study, a minimum reconstruction width of 3 to $5 \mathrm{~mm}$ was required, whereas this was no more than $1.25 \mathrm{~mm}$ in the other studies. ${ }^{11,12}$ Reconstruction width in chest CT scans of 214 of 316 patients $(67.7 \%)$ in our cohort was not more than $3 \mathrm{~mm}$, but only 77 (24.4\%) had a reconstruction width of not more than $1.25 \mathrm{~mm}$. Thinner slice thickness may have resulted in the identification of a higher number of small nodules. Because of continuous technical improvement of CT units, the incidence of small lung nodules might artificially increase in the next studies. Based on the results of the current analysis, one could argue that performing a fine-cut CT of the lungs in patients with RMS has no added value; however, the current EpSSG definition for pulmonary metastases also incorporates patients with five or more small nodules for which a fine-cut CT scan is required.

Although indeterminate pulmonary nodules are a frequent finding in (otherwise) healthy children, finding indeterminate pulmonary nodules in patients with newly diagnosed RMS is more complicated. Histopathologic examination is considered the gold standard for final characterization of these nodules; however, it generally requires surgical biopsy by thoracic surgery, with the chance of

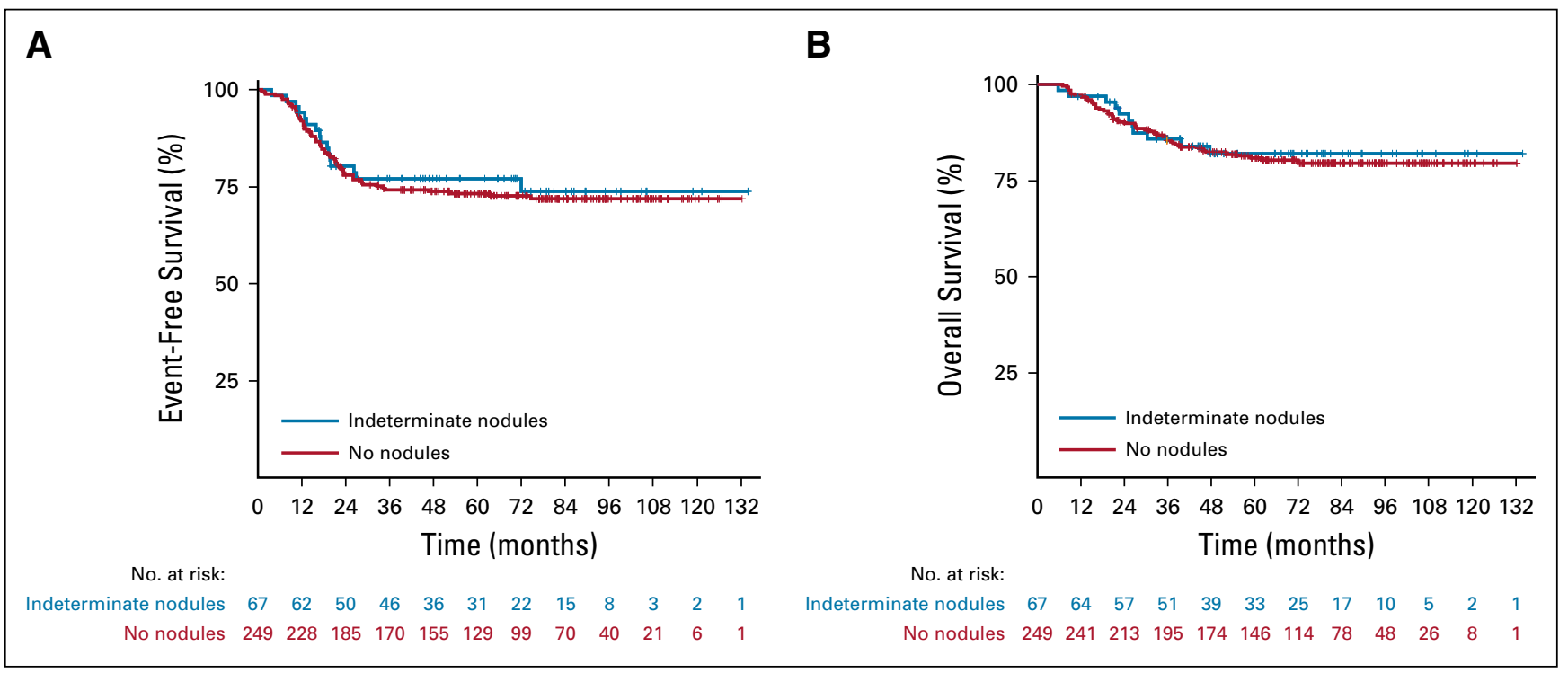

FIG 2. Kaplan-Meier survival curves showing (A) event-free survival and (B) overall survival for patients based on the presence of indeterminate pulmonary nodules at diagnosis. 
TABLE 3. EFS and OS, Based on Number and Size of Nodules at Diagnosis

No.

5-Year EFS (95\% Cl)
EFS $P^{*}$

5-Year OS $(95 \% \mathrm{CI})$

OS $P^{*}$

No. of nodules

.79

\begin{tabular}{lrl}
\hline 0 & 249 & $73.2(67.1$ to 78.3$)$ \\
\hline 1 & 46 & $75.4(60.01$ to 85.6$)$ \\
\hline$>1$ & 21 & $80.2(55.41$ to 92.1$)$
\end{tabular}

Size of largest nodule, $\mathrm{mm}$

$<3$

37

75.3 (57.91 to 86.3)

.74

$\geq 3$

30

79.2 (59.41 to 90.1 )
80.8 (75.11 to 85.3$)$

81.5 (66.41 to 90.3)

81.8 (51.91 to 94.0$)$

82.7 (65.41 to 91.8)

80.7 (59.21 to 91.6)

Abbreviations: EFS, event-free survival; OS, overall survival.

*Based on log-rank test.

false-negative results on examination of biopsy specimens. This strategy was not considered acceptable by the protocol committee of the EpSSG. Therefore, the final decision to upstage patients with indeterminate pulmonary nodules, leading to intensification of standard chemotherapy, and surgery and/or radiotherapy for the pulmonary nodules, was generally based on the assessment of the chest CT scans by pediatric radiologists in collaboration with involved clinicians in tumor board meetings. Radiologists use several parameters to try to distinguish benign from malignant lung nodules; however, none of these parameters have proven to reliably differentiate these nodules. ${ }^{9,10,17}$ Silva et $\mathrm{al}^{10}$ evaluated chest CT scans of 488 children with extrapulmonary malignancies. Of the 488 children, 111 $(22.7 \%)$ had pulmonary nodules at diagnosis; 27 patients also underwent a biopsy and none of the CT characteristics assessed (eg, number and size of nodules) reliably differentiated benign from malignant nodules. McCarville et $\mathrm{al}^{9}$ assessed the chest CT scans of 41 children with malignant solid tumors in whom pulmonary nodules were biopsied (81 nodules in total) and found that small pulmonary nodules (ie, less than $5 \mathrm{~mm}$ ) were as likely to be malignant as larger nodules.

TABLE 4. Type of Event, Based on Presence of Indeterminate Pulmonary Nodules

\begin{tabular}{|c|c|c|c|c|}
\hline \multirow[b]{2}{*}{ Characteristic } & \multicolumn{2}{|c|}{$\begin{array}{l}\text { No Nodule } \\
(n=249)\end{array}$} & \multicolumn{2}{|c|}{$\begin{array}{c}\text { Indeterminate } \\
\text { Pulmonary } \\
\text { Nodule } \\
(\mathrm{n}=67)\end{array}$} \\
\hline & No. & $\%$ & No. & $\%$ \\
\hline \multicolumn{5}{|l|}{ Type of event } \\
\hline Local recurrence & 53 & 21 & 11 & 16 \\
\hline Metastatic recurrence & 5 & 2 & 3 & 4 \\
\hline Local and metastatic recurrence & 6 & 2 & 1 & 1 \\
\hline Second primary malignancy & 3 & 1 & 1 & 1 \\
\hline \multicolumn{5}{|l|}{ Metastatic site } \\
\hline Lung & 4 & 2 & 2 & 3 \\
\hline Other & 7 & 3 & 2 & 3 \\
\hline
\end{tabular}

Because of this limitation, radiologists and pediatric oncologists of the EpSSG established an arbitrary CT definition of stage IV lung disease, based on number and size of nodules, to be used as a noninclusion criterion in the EpSSG RMS 2005 study. Patients with other small pulmonary nodules ( $\leq$ four nodules $<5 \mathrm{~mm}$ or one nodule measuring $\geq 5 \mathrm{~mm}$ and $<10 \mathrm{~mm}$ ) were classified as "indeterminate nodules" and were treated according to localized disease protocol.

The results of the current analysis justify the use of this definition. They illustrate that the presence of these very small indeterminate pulmonary nodules does not affect survival, implying that there is no need to intensify treatment (ie, chest radiotherapy, longer period of maintenance therapy, or other treatment intensification) for these patients in future protocols. Previous studies of patients with lung-only metastatic RMS indicated that survival was affected by histology, age at diagnosis, and the intensity of therapy. ${ }^{4,15,16}$ We found no evidence that these factors influenced our finding that indeterminate pulmonary nodules do not affect survival in RMS, although numbers are limited.

The clinical significance of small pulmonary nodules has previously been assessed in other pediatric malignancies; however, the definition of small pulmonary nodules and the results were inconsistent. Absalon et al $^{17}$ included 210 newly diagnosed patients with bone or soft tissue sarcoma and found pulmonary nodules (diameter $\leq 2 \mathrm{~cm}$ ) in 66 patients (median size of nodules was $5 \mathrm{~mm}$; range, 1 to $20 \mathrm{~mm}$ ). The size of pulmonary nodules was not significantly associated with outcome; however, the number and distribution of nodules was. The same conclusion was drawn by Cipriano et $\mathrm{al}^{18}$ in a retrospective, single-center analysis of 126 patients with high-grade bone or soft tissue sarcoma in which survival was significantly decreased in patients with multiple nodules not larger than $5 \mathrm{~mm}$ and patients with multiple bilateral nodules. Both studies included patients with several histologic diagnoses in whom treatment also differed based on the diagnostic assessments. 
In contrast, patients included in our analysis all had RMS and were uniformly and prospectively treated according to one study protocol. Both patient groups (ie, with and without indeterminate pulmonary nodules) were stratified as having localized disease, allowing us to compare survival between both groups. Although the EpSSG RMS 2005 protocol clearly stated that patients with indeterminate pulmonary nodules should be treated as having localized disease, a small subset of patients underwent a surgical biopsy at diagnosis. We excluded those patients from our analysis; inclusion would have introduced bias because only tumor-negative biopsy specimens $(n=2)$ would have been included in the EpSSG RMS 2005 study for localized disease.

A standardized radiology reporting template was not used in the EpSSG RMS 2005 study and the definition of indeterminate pulmonary nodules was an arbitrary cutoff, we therefore expected an underestimation of reported incidence of indeterminate pulmonary nodules in the radiology reports. This was confirmed by the difference in incidence between initial reports and the reviewed imaging (incidence was more than 10\% higher in reviewed imaging).

The strength of this study is that chest CT scans were reviewed by local pediatric radiologists using a standardized case-report form. Furthermore, this analysis is based on a large cohort of consecutive patients treated according to the same treatment protocol with adequate follow-up. Limitations were that we only included large centers participating in the EpSSG RMS 2005 study, and 55 of 376 potential patients were excluded because the chest CT scan at diagnosis was not available for review. The current cohort $(n=316)$ contained relatively more high-risk patients and patients with higher IRS groups. The participating centers are often international referral centers, which might explain the higher incidence of high-risk patients. Another limitation is that we did not use central

\author{
AFFILIATIONS \\ ${ }^{1}$ University of Amsterdam, Amsterdam, the Netherlands \\ 2Padova University Hospital, Padova, Italy \\ ${ }^{3}$ Great Ormond Street Hospital for Children, London, United Kingdom \\ ${ }^{4}$ Institut Curie, Paris, France \\ ${ }^{5}$ Istituto Nazionale Tumori Milano, Milan, Italy \\ ${ }^{6}$ Centre Léon Bérard, Lyon, France \\ ${ }^{7}$ Children's Hospital for Wales, Heath Park, Cardiff, United Kingdom \\ ${ }^{8}$ Royal Marsden Hospital, Sutton, United Kingdom \\ ${ }^{9}$ Istituto Oncologico Veneto, Padova, Italy \\ ${ }^{10}$ Princess Máxima Center for Pediatric Oncology, Utrecht, the \\ Netherlands
}

\section{CORRESPONDING AUTHOR}

Johannes H.M. Merks, MD, PhD, Princess Máxima Center for Pediatric Oncology, Heidelberglaan 25, 3584 CS, Utrecht, the Netherlands; e-mail: j.h.m.merks@prinsesmaximacentrum.nl. review, because previous studies demonstrated substantial interobserver variability in the detection of pulmonary nodules, more specifically in the detection of smaller nodules. ${ }^{12,19,20}$ A central review of chest CT images could have led to more consistent assessments and reporting. However, this was not possible for organizational reasons; review of chest CT scans by local radiologists was in compliance with the informed consent of the EpSSG RMS 2005 study, whereas central review would have caused regulatory issues. We tried to limit the bias by using a standardized case-report form; nevertheless, this did not exclude interobserver variability.

Another limitation is that we did not assess the CT pattern changes during chemotherapy nor the histology of residual nodules removed after chemotherapy. Nodules that decrease in size or disappear more likely, intuitively, represent micrometastases, whereas unchanged nodules more likely represent benign lesions.

To conclude, in this study, we demonstrated that the presence of indeterminate pulmonary nodules, as defined in the EpSSG RMS 2005 protocol, in patients with newly diagnosed RMS treated for localized disease does not affect survival, implying that patients with indeterminate pulmonary nodules were adequately treated according to the nonmetastatic disease protocol in the EpSSG RMS 2005 study. Importantly, this study indicates that patients with indeterminate pulmonary nodules do not require chest radiotherapy, therewith limiting potential toxicity for these patients. ${ }^{21}$

For future studies, we emphasize the importance of standardized imaging-reporting templates to improve consistency of reporting. The new International Society of Pediatric Oncology-Quality and Excellence in Radiotherapy and Imaging for Children and Adolescents with Cancer across Europe initiative could contribute to this. ${ }^{22}$

\section{PRIOR PRESENTATION}

Presented at the European Society of Pediatric Radiology annual meeting, Berlin, Germany, June 18-22, 2018; and at the International Society of Pediatric Oncology Congress, Kyoto, Japan, November 16-19, 2018.

\section{SUPPORT}

Supported by Foundation KiKa. J.C.C. was supported by National Health Service funding to the National Institute for Health Research Biomedical Research Center of the Royal Marsden Hospital. The RMS2005 study has been supported by Fondazione Città della Speranza, Italy. These foundations had no role in study design or interpretation of the data.

AUTHOR'S DISCLOSURES OF POTENTIAL CONFLICTS OF INTEREST AND DATA AVAILABILITY STATEMENT

Disclosures provided by the author and data availability statement (if applicable) are available with this article at DOI https://doi.org/10.1200/ JC0.18.01535. 
AUTHOR CONTRIBUTIONS

Conception and design: Bas Vaarwerk, Gianni Bisogno, Kieran McHugh, Meriel Jenney, Andrea Ferrari, Rick R. van Rijn, Johannes H.M. Merks Administrative support: Bas Vaarwerk, Gianni Bisogno, Johannes H.M. Merks

Provision of study material or patients: Bas Vaarwerk, Gianni Bisogno, Hervé J. Brisse, Daniel Orbach, Julia C. Chisholm, Andrea Ferrari, Johannes H.M. Merks

Collection and assembly of data: Bas Vaarwerk, Gianni Bisogno, Kieran McHugh, Hervé J. Brisse, Carlo Morosi, Nadège Corradini, Daniel
Orbach, Julia C. Chisholm, Andrea Ferrari, Gian Luca De Salvo, Johannes H.M. Merks

Data analysis and interpretation: Gianni Bisogno, Kieran McHugh, Meriel Jenney, Daniel Orbach, Ilaria Zanetti, Gian Luca De Salvo, Rick R. van Rijn, Johannes H.M. Merks

Manuscript writing: All authors

Final approval of manuscript: All authors

Accountable for all aspects of the work: All authors

\section{REFERENCES}

1. Oberlin O, Rey A, Sanchez de Toledo J, et al: Randomized comparison of intensified six-drug versus standard three-drug chemotherapy for high-risk nonmetastatic rhabdomyosarcoma and other chemotherapy-sensitive childhood soft tissue sarcomas: Long-term results from the International Society of Pediatric Oncology MMT95 study. J Clin Oncol 30:2457-2465, 2012

2. Arndt CA, Stoner JA, Hawkins DS, et al: Vincristine, actinomycin, and cyclophosphamide compared with vincristine, actinomycin, and cyclophosphamide alternating with vincristine, topotecan, and cyclophosphamide for intermediate-risk rhabdomyosarcoma: Children's Oncology Group study D9803. J Clin Oncol 27:5182-5188, 2009

3. Bisogno G, Jenney M, Bergeron C, et al: Addition of dose-intensified doxorubicin to standard chemotherapy for rhabdomyosarcoma (EpSSG RMS 2005): A multicentre, open-label, randomised controlled, phase 3 trial. Lancet Oncol 19:1061-1071, 2018

4. Oberlin O, Rey A, Lyden E, et al: Prognostic factors in metastatic rhabdomyosarcomas: Results of a pooled analysis from United States and European cooperative groups. J Clin Oncol 26:2384-2389, 2008

5. Weigel BJ, Lyden E, Anderson JR, et al: Intensive multiagent therapy, including dose-compressed cycles of ifosfamide/etoposide and vincristine/doxorubicin/ cyclophosphamide, irinotecan, and radiation, in patients with high-risk rhabdomyosarcoma: A report from the Children's Oncology Group. J Clin Oncol 34: 117-122, 2016

6. Picci P, Vanel D, Briccoli A, et al: Computed tomography of pulmonary metastases from osteosarcoma: The less poor technique. A study of 51 patients with histological correlation. Ann Oncol 12:1601-1604, 2001

7. Brader P, Abramson SJ, Price AP, et al: Do characteristics of pulmonary nodules on computed tomography in children with known osteosarcoma help distinguish whether the nodules are malignant or benign? J Pediatr Surg 46:729-735, 2011 [Erratum: J Pediatr Surg 2011;46:1685]

8. Grampp S, Bankier AA, Zoubek A, et al: Spiral CT of the lung in children with malignant extra-thoracic tumors: Distribution of benign vs malignant pulmonary nodules. Eur Radiol 10:1318-1322, 2000

9. McCarville MB, Lederman HM, Santana VM, et al: Distinguishing benign from malignant pulmonary nodules with helical chest CT in children with malignant solid tumors. Radiology 239:514-520, 2006

10. Silva CT, Amaral JG, Moineddin R, et al: CT characteristics of lung nodules present at diagnosis of extrapulmonary malignancy in children. AJR Am J Roentgenol 194:772-778, 2010

11. Renne J, Linderkamp C, Wacker F, et al: Prevalence and configuration of pulmonary nodules on multi-row CT in children without malignant diseases. Eur Radiol 25:2651-2656, 2015

12. Samim A, Littooij AS, van den Heuvel-Eibrink MM, et al: Frequency and characteristics of pulmonary nodules in children at computed tomography. Pediatr Radiol 47:1751-1758, 2017

13. Gallego S, Zanetti I, Orbach D, et al: Fusion status in patients with lymph node-positive (N1) alveolar rhabdomyosarcoma is a powerful predictor of prognosis: Experience of the European Paediatric Soft Tissue Sarcoma Study Group (EpSSG). Cancer 124:3201-3209, 2018

14. Kaplan EL, Meier P: Nonparametric estimation from incomplete observations. J Am Stat Assoc 53:457-481, 1958

15. Rodeberg D, Arndt C, Breneman J, et al: Characteristics and outcomes of rhabdomyosarcoma patients with isolated lung metastases from IRS-IV. J Pediatr Surg 40:256-262, 2005

16. Sparber-Sauer M, von Kalle T, Seitz G, et al: The prognostic value of early radiographic response in children and adolescents with embryonal rhabdomyosarcoma stage IV, metastases confined to the lungs: A report from the Cooperative Weichteilsarkom Studiengruppe (CWS). Pediatr Blood Cancer 64: e26510, 2017

17. Absalon MJ, McCarville MB, Liu T, et al: Pulmonary nodules discovered during the initial evaluation of pediatric patients with bone and soft-tissue sarcoma. Pediatr Blood Cancer 50:1147-1153, 2008

18. Cipriano C, Brockman L, Romancik J, et al: The clinical significance of initial pulmonary micronodules in young sarcoma patients. J Pediatr Hematol Oncol 37 : 548-553, 2015

19. Kilburn-Toppin F, Arthurs OJ, Tasker AD, et al: Detection of pulmonary nodules at paediatric CT: Maximum intensity projections and axial source images are complementary. Pediatr Radiol 43:820-826, 2013

20. Wilimas JA, Kaste SC, Kauffman WM, et al: Use of chest computed tomography in the staging of pediatric Wilms' tumor: Interobserver variability and prognostic significance. J Clin Oncol 15:2631-2635, 1997

21. Stoppel G, Eich HT, Matuschek C, et al: Lung toxicity after radiation in childhood: Results of the International Project on Prospective Analysis of Radiotoxicity in Childhood and Adolescence. Radiother Oncol 125:286-292, 2017

22. Vassal G, Schrappe M, Pritchard-Jones K, et al: The SIOPE strategic plan: A European cancer plan for children and adolescents. J Cancer Policy 8:17-32, 2016 
AUTHORS' DISCLOSURES OF POTENTIAL CONFLICTS OF INTEREST

Indeterminate Pulmonary Nodules at Diagnosis in Rhabdomyosarcoma: Are They Clinically Significant? A Report From the European Paediatric Soft Tissue Sarcoma Study Group

The following represents disclosure information provided by authors of this manuscript. All relationships are considered compensated. Relationships are self-held unless noted. I = Immediate Family Member, Inst = My Institution. Relationships may not relate to the subject matter of this manuscript. For more information about ASCO's conflict of interest policy, please refer to www.asco.org/rwc or ascopubs.org/jco/site/ifc.

\section{Gianni Bisogno}

Consulting or Advisory Role: Clinigen Group, Roche

Speakers' Bureau: Merck

Travel, Accommodations, Expenses: Jazz Pharmaceuticals

Daniel Orbach

Consulting or Advisory Role: Bayer

Julia C. Chisholm

Consulting or Advisory Role: Roche, Bayer

\section{Gian Luca De Salvo}

Travel, Accommodations, Expenses: Bayer

Rick R. van Rijn

Research Funding: Roche Pharma (Inst)

No other potential conflicts of interest were reported. 
The European Soft Tissue Sarcoma Study Group radiology group members all participated as contributors to this study. The group consisted of:

Amine Bouhamama, Centre Léon Bérard, Lyon France.

Shruti Moholkar, Birmingham Children's Hospital, Birmingham, UK.

Susan Morris, Children's Hospital for Wales, Heath Park, Cardiff, UK.

Giovanna Orsatti, University Hospital of Padova, Padova, Italy.

Erika Pace, Royal Marsden Hospital, Sutton, UK.

Rui M.F. Santos, Royal Manchester Children's Hospital, Manchester, UK.

Joost van Schuppen, Beatrix Children's Hospital, University Medical Center Groningen, Groningen, the Netherlands.

Ewan Simpson, Bristol Royal Hospital for Children, Bristol, UK. 